\title{
Higher Plasma Concentrations of the Methylglyoxal Metabolite D-lactate are Independently Associated with Insulin Resistance: The CODAM Study
}

Dionne EM Maessen ${ }^{1,2}$, Jean LJM Scheijen ${ }^{1,2}$, Katrien H Gaens ${ }^{1,2}$, Marleen MJ van Greevenbroek ${ }^{1,2}$, Carla J van der Kallen ${ }^{1,2}$, Coen DA Stehouwer ${ }^{1,2}$ and Casper G Schalkwijk $\mathbf{k}^{1,2 *}$

${ }^{1}$ CARIM School for Cardiovascular Diseases, Maastricht University Medical Centre, Maastricht, The Netherlands

${ }^{2}$ Department of Internal Medicine, Maastricht University Medical Centre, Maastricht, The Netherlands

\begin{abstract}
Objective: Methylglyoxal (MGO), a highly reactive dicarbonyl compound generated by the spontaneous degradation of glycolytic intermediates, is a major precursor for advanced glycation endproducts and can potentially disrupt cellular functions. MGO can be detoxified by the glyoxalase system into D-lactate. Although experimental studies have shown that increased levels of MGO are associated with insulin resistance, epidemiological evidence of such an association in human studies is lacking. The aim of this study was to investigate the association between plasma D-lactate, as a reflection of plasma MGO concentrations, and insulin resistance.

Methods: Cross-sectional, complete case analyses were performed in the Cohort study on Diabetes and Atherosclerosis Maastricht (CODAM). 513 participants were included in the study population: $59.4 \pm 6.9$ years of age, 63\% men, and, by design, 23\% impaired glucose metabolism (IGM) and 23\% type 2 diabetes mellitus (T2DM). Plasma D-lactate was measured by UPLC-MS/MS. The main outcome measure was HOMA2-IR, as a measure of insulin resistance. The association between plasma D-lactate and HOMA2-IR was studied by multiple linear regression analysis.

Results: The prevalence of T2DM increased significantly over the tertiles of plasma D-lactate concentrations. Moreover, plasma D-lactate was positively associated with HOMA2-IR, when adjusted for age and sex $(\beta=0.429 ; 95 \%$ $\mathrm{Cl}: 0.350-0.507 ; p<0.001$ ), as well as in the fully adjusted model, additionally adjusted for glucose metabolism status, smoking status, prior cardiovascular disease, use of medication (glucose-, lipid-, and blood pressure lowering), estimated glomerular filtration rate, waist circumference, glycated hemoglobin $\left(\mathrm{HbA}_{1 \mathrm{c}}\right)$ and plasma L-lactate levels ( $\beta=0.145 ; 95 \% \mathrm{Cl}$ : $0.051-0.239 ; p=0.003$ ). Additional data indicate that other sources of $D$-lactate can be excluded.

Conclusion: We found a positive association between plasma D-lactate and HOMA2-IR, independently of putative confounders. These results suggest that MGO plays a role in insulin resistance, although direct measurement of MGO is necessary to confirm this.
\end{abstract}

Keywords: D-lactate; Methylglyoxal; Glyoxalase; Carbonyl stress; Insulin resistance; Diabetes; Cohort study

Abbreviations: AGE: Advanced Glycation Endproduct; BP: Blood Pressure; CKD-EPI: Chronic Kidney Disease Epidemiology Collaboration; CODAM: Cohort study on Diabetes and Atherosclerosis Maastricht; CVD: cardiovascular disease; eGFR: estimated Glomerular Filtration Rate; GLO: Glyoxalase; $\mathrm{HbA}_{1 \mathrm{C}}$ : glycated hemoglobin; HOMA2-IR: Homeostasis Model of Assessment - Insulin Resistance; IBD: Inflammatory Bowel Disease; IGM: Impaired Glucose Metabolism; MGO: Methylglyoxal; PEG: Polyethylene Glycol; T2DM: Type 2 Diabetes Mellitus

\section{Introduction}

Insulin resistance with respect to glucose metabolism, is a metabolic condition defined by a reduced responsiveness to the action of insulin on glucose uptake, metabolism or storage [1]. Recently, increased levels of advanced glycation endproducts (AGEs) have been found in association with insulin resistance [1-3].

AGEs are a family of unavoidable by-products of various metabolic pathways. Methylglyoxal (MGO) has received considerable attention as the most reactive and potent AGE precursor [4,5]. MGO is a dicarbonyl compound generated by the spontaneous degradation of glycolytic intermediates during glycolysis. Under physiological circumstances, MGO can be detoxified by the glyoxalase (GLO) system. This system consists of two enzymes, glyoxalase 1 (GLO-1) and glyoxalase 2 (GLO2), which catalyze the conversion of MGO to D-lactate [6].
Although several in vitro experiments and animal studies have indicated a substantial role for MGO in the induction of insulin resistance [1,7], the association between MGO and insulin resistance in humans has not yet been studied. However, because of the high reactivity of MGO with plasma proteins, it is difficult to obtain valid and precise measurements of MGO in plasma. Since MGO is metabolized to D-lactate, we used plasma D-lactate levels as a reflection of MGO concentrations in the current analysis [8]. We previously demonstrated that we are able to measure D-lactate in a very specific way that can efficiently distinguish between D-lactate and L-lactate [9]. MGO degradation is the major route of D-lactate formation, although D-lactate can also originate from other sources such as the intestines.

*Corresponding author: Casper G Schalkwijk, Department of Internal Medicine, Laboratory for Metabolism and Vascular Medicine, Maastricht University Medical Centre, Peter Debeyelaan 25, P.O. Box 5800, 6202 AZ Maastricht, The Netherlands, Tel: +31 433882186; Fax: +31 433875006 E-mail: C.Schalkwijk@maastrichtuniversity.nl

Received June 17, 2014; Accepted October 24, 2014; Published October 26 , 2014

Citation: Maessen DEM, Scheijen JLJM, Gaens KH, van Greevenbroek MMJ, van der Kallen CJ, et al. (2014) Higher Plasma Concentrations of the Methylglyoxal Metabolite D-lactate are Independently Associated with Insulin Resistance: The CODAM Study. J Diabetes Metab 5: 457 doi:10.4172/2155-6156.1000457

Copyright: (C) 2014 Maessen DEM, et al. This is an open-access article distributed under the terms of the Creative Commons Attribution License, which permits unrestricted use, distribution, and reproduction in any medium, provided the original author and source are credited. 
In previous work, however, we found that plasma levels of D-lactate were significantly increased in patients with type 2 diabetes mellitus (T2DM) compared to healthy controls, whereas patients suffering from inflammatory bowel disease (IBD) did not show increased levels of plasma D-lactate [9]. These data underline that increased concentrations of plasma D-lactate are derived from MGO and are not a reflection of $\mathrm{D}$-lactate produced by the intestines.

In this study, we investigated if plasma D-lactate, as a reflection of MGO concentrations, is increased in individuals with impaired glucose metabolism (IGM) and T2DM, and whether plasma D-lactate is associated with insulin resistance in a cohort study designed to investigate glucose metabolism and cardiovascular disease (CVD).

\section{Materials and Methods}

\section{Study population}

The study population consisted of participants from the Cohort study on Diabetes and Atherosclerosis Maastricht (CODAM). This study included 574 participants who were selected from the general population, as described in detail elsewhere [10]. The cohort was enriched for impaired glucose metabolism status, as defined previously [11]. Current analyses were performed on participants who were not on insulin treatment and had complete data on all variables of interest (plasma D-lactate, HOMA2-IR, age, sex, glucose metabolism status, smoking status, prior CVD, use of medication (glucose-, lipid-, and blood pressure-lowering), estimated glomerular filtration rate (eGFR), waist circumference, glycated hemoglobin $\left(\mathrm{HbA}_{1 \mathrm{C}}\right)$ and plasma L-lactate levels). Hence, the present study reports on 513 persons. The study protocol was approved by the local Medical Ethical Committee of Maastricht University Medical Centre and written informed consent was obtained from all participants.

\section{Measurement of plasma D-lactate}

Plasma D-lactate levels were measured in EDTA plasma samples from overnight fasting venous blood. Samples were analyzed by reversed phase ultra-performance liquid chromatography tandem mass spectrometry (UPLC-MS/MS), as described in detail previously [9]. The intra- and inter-assay variations were 4 and $8 \%$, respectively.

\section{Measurement of insulin resistance}

Insulin resistance was estimated using the Homeostasis Model of Assessment - Insulin Resistance (HOMA2-IR) calculator from www. dtu.ox.ac.uk, as described previously [12].

\section{Measurements of covariates}

Plasma L-lactate levels were measured in EDTA plasma samples from overnight fasting venous blood, using UPLC-MS/MS [9]. It was measured synchronously with plasma D-lactate. Smoking behavior (never, ever or current smoker) was assessed by a questionnaire. Prior CVD and waist circumference were assessed as previously described in detail [13]. Creatinine levels were measured in EDTA plasma using a Jaffe diagnostic test (Roche Diagnostics) and the Chronic Kidney Disease Epidemiology Collaboration (CKD-EPI) equation was used to calculate the eGFR [14]. Finally, $\mathrm{HbA}_{1 \mathrm{C}}$ was measured using ionexchange HPLC [10].

\section{Use of medication}

Subjects were asked to report if they were currently using medication, and, if so, to list all the medication used. All medicines were reviewed with the use of the Dutch Pharmacological Compass 2006 (www.fk.cvz. nl) and allocated into 36 categories. Medication containing the additive polyethylene glycol (PEG) was selected using the database of College ter Beoordeling van Geneesmiddelen (www.dbg-med.nl).

\section{Definition of intestinal disease}

Diseases of study participants were self-reported by questionnaire. Intestinal disease was reported when the subject suffered from any serious intestinal disease for 3 months or longer in the past 12 months.

\section{Statistical analyses}

The general characteristics of the study population were compared across tertiles of plasma D-lactate concentrations. One-way analysis of variance (ANOVA) was used for continuous variables and chisquare tests for discrete variables. Skewed variables (plasma D-lactate, HOMA2-IR, fasting plasma glucose, plasma L-lactate and triglycerides) were compared across the plasma D-lactate tertiles by Kruskal-Wallis one-way analysis of variance and were $\log _{e}$ transformed prior to regression analyses. Multiple linear regression analysis was used to investigate the cross-sectional association between plasma D-lactate and HOMA2-IR and results are presented as standardized regression coefficients $(\beta)$. The analysis was initially adjusted for the covariates age (years) and sex (model 1), additionally adjusted for glucose metabolism status (IGM and T2DM as dummy variables), smoking status (exsmoker and current smoker as dummy variables), prior CVD, use of medication (glucose-, lipid-, and blood pressure-lowering medication) and eGFR (model 2), further adjusted for $\mathrm{HbA}_{1 \mathrm{C}}$ (model 3), and finally fully adjusted for plasma L-lactate levels (model 4). A P-value of $<0.05$ was considered statistically significant. All statistical analyses were performed with IBM SPSS Statistics Software, version 20 (IBM Corporation, Armonk, New York).

\section{Results}

General characteristics of the study population stratified according to tertiles of plasma D-lactate concentrations are shown in Table 1. The prevalence of IGM did not differ between the tertiles of plasma D-lactate levels (Table 1). However, the prevalence of T2DM increased significantly with higher plasma D-lactate levels $(\mathrm{p}<0.001)$ (Table 1). Similar results were found for fasting plasma glucose levels, HOMA2IR, and $\mathrm{HbA}_{1 \mathrm{C}}($ all $\mathrm{p}<0.001)$ (Table 1).

Plasma D-lactate concentrations were positively associated with HOMA2-IR, in the model adjusted for age and sex (Model 1: $\beta=0.429$; 95\% CI: $0.350-0.507 ; \mathrm{p}<0.001$ ), as well as in a model adjusted for the additional covariates glucose metabolism status, smoking status, prior CVD, use of medication, eGFR and waist circumference (Model 2: $\beta=0.214$; 95\% CI: 0.147-0.280; $p<0.001$ ). After additional adjustment for $\mathrm{HbA}_{1 \mathrm{C}}$, which had a negligible effect on the regression coefficient, plasma D-lactate remained positively associated with HOMA2-IR (Model 3: $\beta=0.210 ; 95 \%$ CI: 0.143-0.276; $\mathrm{p}<0.001$ ). Addition of plasma L-lactate levels to model 3 resulted in a relatively large decrease in the regression coefficient (Model 4: $\beta=0.145$; 95\% CI: 0.051-0.239; $\mathrm{p}=0.003)$, although the association between plasma D-lactate and HOMA2-IR remained significant (Table 2).

Although MGO degradation is the major route of D-lactate formation, D-lactate can also be produced by other pathways. We already found in our previous study that patients suffering from IBD did not show increased levels of plasma D-lactate. However, we additionally analyzed our study population with regard to intestinal disease. There were no differences in prevalence of intestinal disease between the plasma D-lactate tertiles (T1: $1.8 \%$, T2: $4.7 \%$, T3: $2.9 \%$; 
Citation: Maessen DEM, Scheijen JLJM, Gaens KH, van Greevenbroek MMJ, van der Kallen CJ, et al. (2014) Higher Plasma Concentrations of the Methylglyoxal Metabolite D-lactate are Independently Associated with Insulin Resistance: The CODAM Study. J Diabetes Metab 5: 457 doi:10.4172/2155-6156.1000457

Page 3 of 4

\begin{tabular}{|c|c|c|c|c|}
\hline & \multicolumn{3}{|c|}{ Plasma D-lactate tertiles } & \multirow[b]{2}{*}{ P-value } \\
\hline & Lowest $(n=171)$ & Middle $(n=171)$ & Highest $(n=171)$ & \\
\hline Age, years & $59.2 \pm 6.9$ & $59.9 \pm 7.1$ & $59.1 \pm 6.8$ & 0.43 \\
\hline Sex, \% men & 57.3 & 66.1 & 64.9 & 0.19 \\
\hline Body mass index, $\mathrm{kg} / \mathrm{m}^{2}$ & $27.3 \pm 3.7$ & $28.0 \pm 4.1$ & $30.0 \pm 4.4$ & $<0.001$ \\
\hline Waist circumference, cm & $95.5 \pm 10.9$ & $98.6 \pm 11.2$ & $103.5 \pm 12.0$ & $<0.001$ \\
\hline IGM, \% & 23.4 & 20.5 & 25.1 & 0.58 \\
\hline T2DM, \% & 11.7 & 21.6 & 35.1 & $<0.001$ \\
\hline Fasting plasma glucose, $\mathrm{mmol} / \mathrm{L}$ & $5.4[5.0-5.8]$ & $5.6[5.2-6.1]$ & $5.9[5.4-7.1]$ & $<0.001$ \\
\hline HOMA2-IR & $0.9[0.7-1.3]$ & $1.2[0.9-1.6]$ & $1.4[1.0-2.3]$ & $<0.001$ \\
\hline Plasma D-lactate, $\mu \mathrm{mol} / \mathrm{L}$ & $5.5[4.6-6.3]$ & $8.5[7.8-9.7]$ & $14.5[12.6-17.1]$ & $<0.001$ \\
\hline Plasma L-lactate, $\mu \mathrm{mol} / \mathrm{L}$ & 922 [795-1054] & $1143[974-1315]$ & $1634[1358-1884]$ & $<0.001$ \\
\hline $\mathrm{HbA}_{1 \mathrm{c}}, \%$ & $5.7 \pm 0.7$ & $5.9 \pm 0.7$ & $6.1 \pm 0.8$ & $<0.001$ \\
\hline Glucose-lowering medication, \% & 5.3 & 9.4 & 17.0 & 0.002 \\
\hline Prior CVD, \% & 21.6 & 26.9 & 32.7 & 0.07 \\
\hline Total cholesterol, mmol/L & $4.2 \pm 1.3$ & $4.8 \pm 1.6$ & $5.2 \pm 1.7$ & $<0.001$ \\
\hline Triglycerides, mmol/L & $1.1[0.8-1.5]$ & $1.4[1.0-1.9]$ & $1.8[1.4-2.4]$ & $<0.001$ \\
\hline Lipid-lowering medication, \% & 15.8 & 17.0 & 20.5 & 0.50 \\
\hline Current smoker, \% & 20.5 & 19.3 & 22.8 & 0.72 \\
\hline Former smoker, \% & 52.0 & 51.5 & 51.5 & 0.99 \\
\hline BP-lowering medication, \% & 28.7 & 38.0 & 46.2 & 0.004 \\
\hline eGFR, $\mathrm{mL} / \mathrm{min} / 1.73 \mathrm{~m}^{2}$ & $90.5 \pm 11.5$ & $90.2 \pm 12.6$ & $93.8 \pm 12.1$ & 0.009 \\
\hline
\end{tabular}

Data are presented as means $\pm S D$, medians (interquartile range), or percentages, as appropriate; the ranges of the plasma $D$-lactate tertiles were [2.6-7.1], [7.1-10.7] and [10.7-32.4] $\mu \mathrm{mol} / \mathrm{L}$ respectively. IGM: impaired glucose metabolism; T2DM: type 2 diabetes mellitus; HOMA2-IR: homeostasis model assessment insulin resistance; HbA 1 : glycated hemoglobin; CVD: cardiovascular disease; BP: blood pressure; eGFR: estimated glomerular filtration rate.

Table 1: General characteristics of the study population stratified according to tertiles of plasma D-lactate.

\begin{tabular}{|c|c|c|c|}
\hline Dependent variable & Model & $\boldsymbol{\beta}$ & $\mathbf{9 5 \%} \mathbf{C l}$ \\
\hline \multirow{3}{*}{ HOMA2-IR } & 1 & 0.429 & $0.350-0.507$ \\
\cline { 2 - 4 } & 2 & 0.214 & $0.147-0.280$ \\
\cline { 2 - 4 } & 3 & 0.210 & $0.143-0.276$ \\
\hline
\end{tabular}

The standardized regression coefficient $\beta$ represents one standard deviation (SD) increase in HOMA2-IR per SD increase in plasma D-lactate.

Model 1: adjusted for age and sex;

Model 2: model 1 + glucose metabolism status, smoking status, prior CVD, use of medication (glucose-, lipid-, and blood pressure lowering), estimated glomerular filtration rate and waist circumference;

Model 3: model $2+\mathrm{HbA}_{1 \mathrm{c}}$

Model 4: model $3+$ plasma L-lactate levels.

Table 2: Association between plasma D-lactate $(\mu \mathrm{mol} / \mathrm{L})$ and HOMA2-IR

$\mathrm{p}=0.29$ ), nor was there a difference in the proportion of intestinal diseased participants treated for this disease (T1: 50\%, T2: 57\%, T3: $44 \% ; \mathrm{p}=0.88$ )

In addition to the intestinal route, D-lactate can also be formed from PEG, which is used as an additive for several medications. To exclude PEG as a source of D-lactate production, we selected all medication in our cohort which was enriched with PEG, and analyzed whether there were more users of PEG-contaminated medication in the higher tertile of plasma D-lactate. We found that the users of PEG-contaminated medication were equally distributed between the tertiles of D-lactate (T1: 35\%, T2: 40\%, T3: 46\%; $\mathrm{p}=0.14$ ). Since PEG is often added to BPlowering medication, we performed the same analyses for this specific group of medication. Again, no difference in amount of users was found between the D-lactate tertiles (T1: 57\%, T2: 66\%, T3: 60\%; $\mathrm{p}=0.58$ ).

\section{Discussion}

In this cohort study we demonstrated that plasma D-lactate levels are increased in patients with T2DM. Additionally, we found a positive association between plasma D-lactate levels and HOMA2-IR, independently of putative confounders.
This is the first study that has examined the association between MGO and insulin resistance in a large human cohort study, using plasma D-lactate as a reflection of MGO concentrations. We observed a significantly higher prevalence of T2DM across the tertiles of plasma D-lactate concentrations. A similar trend was observed for fasting plasma glucose levels, $\mathrm{HOMA} 2-\mathrm{IR}$, and $\mathrm{HbA}_{1 \mathrm{C}}$, supporting the hypothesis that persons with abnormal glucose metabolism have high plasma D-lactate levels. These positive associations are in line with our previous study, in which we demonstrated that T2DM patients have increased plasma and urine levels of D-lactate [9] and with studies showing that the concentration of D-lactate typically increased in cells cultured in high glucose concentrations $[5,8]$.

We found a strong association between plasma D-lactate and HOMA2-IR, which was independent of confounders. Although adjustment for plasma L-lactate levels resulted in a relatively large decrease in the regression coefficient, the association between plasma D-lactate and HOMA2-IR remained significant, demonstrating an association between plasma D-lactate probably derived from MGO metabolism and insulin resistance. Our results are in agreement with several experimental studies showing that the major AGE-precursor 
Citation: Maessen DEM, Scheijen JLJM, Gaens KH, van Greevenbroek MMJ, van der Kallen CJ, et al. (2014) Higher Plasma Concentrations of the Methylglyoxal Metabolite D-lactate are Independently Associated with Insulin Resistance: The CODAM Study. J Diabetes Metab 5: 457 doi:10.4172/2155-6156.1000457

Page 4 of 4

MGO potentially contributes to the development of insulin resistance. It has been demonstrated that administration of MGO to rats caused impaired insulin signaling and induced insulin resistance [15-18]. In addition, chronic infusion of MGO caused $\beta$-cell dysfunction [18]. Next to the in vivo studies, in vitro experiments with 3T3 adipocytes and INS-1E pancreatic cells showed that IRS-1 phosphorylation and PI3K activity were dose-dependently reduced by treatment with MGO, indicating its contribution to the pathogenesis of insulin resistance $[17,19]$.

Our finding that plasma D-lactate is positively and independently associated with insulin resistance is in line with findings from recent studies, which described a positive association between AGEs and insulin resistance [1-3]. However, we cannot establish whether these associations are causal due to the cross-sectional design of the study. Further follow-up data on these or other patient groups would, of course, be invaluable in testing whether our findings are probably due to causal relationships between MGO and insulin resistance. However, this cross-sectional study may serve as a reasonable starting point to further explore these associations. Plasma D-lactate may be, as a reflection of plasma MGO, an early marker of insulin resistance.

The main limitation of our study is that we were not able so far to measure MGO directly in these plasma samples. However, it is very likely that an increase in D-lactate concentrations is indicative of increased flux of MGO formation, as was demonstrated in erythrocytes [8]. Furthermore, our additional analyses showed that we can exclude other sources than MGO for the production of D-lactate. Previous work and current results indicate that both the intestines as well as PEGcontaminated medication do not contribute to plasma D-lactate levels.

In summary, our data show for the first time that plasma D-lactate levels are associated with HOMA2-IR, independently of putative confounders. Together with findings from previous studies, our results suggest that higher levels of MGO play an important role in the etiology of insulin resistance, although this has to be confirmed in future research.

\section{References}

1. Schalkwijk CG, Brouwers O, Stehouwer CD (2008) Modulation of insulin action by advanced glycation endproducts: a new player in the field. Horm Metab Res 40: $614-619$

2. Sandu O, Song K, Cai W, Zheng F, Uribarri J, et al. (2005) Insulin resistance and type 2 diabetes in high-fat-fed mice are linked to high glycotoxin intake. Diabetes 54: 2314-2319.

3. Forbes JM, Sourris KC, de Courten MP, Dougherty SL, Chand V, et al. (2014) Advanced glycation end products (AGEs) are cross-sectionally associated with insulin secretion in healthy subjects. Amino Acids 46: 321-326.

4. Westwood ME, Thornalley PJ (1995) Molecular characteristics of methylglyoxalmodified bovine and human serum albumins. Comparison with glucose-derived advanced glycation endproduct-modified serum albumins. J Protein Chem 14 359-372.

5. Shinohara M, Thornalley PJ, Giardino I, Beisswenger P, Thorpe SR, et al. (1998) Overexpression of glyoxalase-I in bovine endothelial cells inhibits intracellular advanced glycation endproduct formation and prevents hyperglycemia-induced increases in macromolecular endocytosis. J Clin Invest 101: 1142-1147.
6. Thornalley PJ (1993) The glyoxalase system in health and disease. Mol Aspects Med 14: 287-371.

7. Matafome P, Sena C, Seiça R (2013) Methylglyoxal, obesity, and diabetes. Endocrine 43: 472-484.

8. Thornalley PJ (1988) Modification of the glyoxalase system in human red blood cells by glucose in vitro. Biochem J 254: 751-755.

9. Scheijen JL, Hanssen NM, van de Waarenburg MP, Jonkers DM, Stehouwer $C D$, et al. (2012) $L(+)$ and $D(-)$ lactate are increased in plasma and urine samples of type 2 diabetes as measured by a simultaneous quantification of $\mathrm{L}(+)$ and $\mathrm{D}(-)$ lactate by reversed-phase liquid chromatography tandem mass spectrometry. Experimental diabetes research 234812

10. Du H, van der A DL, van Bakel MM, van der Kallen CJ, Blaak EE, et al. (2008) Glycemic index and glycemic load in relation to food and nutrient intake and metabolic risk factors in a Dutch population. Am J Clin Nutr 87: 655-661.

11. Hanssen NM, Engelen L, Ferreira I, Scheijen JL, Huijberts MS, et al. (2013) Plasma levels of advanced glycation endproducts $\mathrm{N} \varepsilon$-(carboxymethyl)lysine $\mathrm{N} \varepsilon$-(carboxyethyl)lysine, and pentosidine are not independently associated with cardiovascular disease in individuals with or without type 2 diabetes: the Hoorn and CODAM studies. J Clin Endocrinol Metab 98: E1369-1373.

12. Wlazlo N, van Greevenbroek MM, Ferreira I, Jansen EH, Feskens EJ, et al (2013) Iron metabolism is associated with adipocyte insulin resistance and plasma adiponectin: the Cohort on Diabetes and Atherosclerosis Maastricht (CODAM) study. Diabetes Care 36: 309-315.

13. van Greevenbroek MM, Jacobs M, van der Kallen CJ, Vermeulen VM, Jansen $\mathrm{EH}$, et al. (2011) The cross-sectional association between insulin resistance and circulating complement $\mathrm{C} 3$ is partly explained by plasma alanine aminotransferase, independent of central obesity and general inflammation (the CODAM study). Eur J Clin Invest 41: 372-379.

14. Levey AS, Stevens LA, Schmid CH, Zhang YL, Castro AF 3rd, et al. (2009) A new equation to estimate glomerular filtration rate. Ann Intern Med 150: 604612.

15. Guo Q, Mori T, Jiang Y, Hu C, Osaki Y, et al. (2009) Methylglyoxal contributes to the development of insulin resistance and salt sensitivity in Sprague-Dawley rats. J Hypertens 27: 1664-1671.

16. Dhar A, Desai KM, Wu L (2010) Alagebrium attenuates acute methylglyoxalinduced glucose intolerance in Sprague-Dawley rats. Br J Pharmacol 159: 166 175.

17. Jia X, Wu L (2007) Accumulation of endogenous methylglyoxal impaired insulin signaling in adipose tissue of fructose-fed rats. Mol Cell Biochem 306: 133-139.

18. Dhar A, Dhar I, Jiang B, Desai KM, Wu L (2011) Chronic methylglyoxal infusion by minipump causes pancreatic beta-cell dysfunction and induces type 2 diabetes in Sprague-Dawley rats. Diabetes 60: 899-908.

19. Fiory F, Lombardi A, Miele C, Giudicelli J, Beguinot F, et al. (2011) Methylglyoxa impairs insulin signalling and insulin action on glucose-induced insulin secretion in the pancreatic beta cell line INS-1E. Diabetologia 54: 2941-2952. 